\title{
Occupational Exposure of Dentists to Extremely-low-frequency Magnetic Field
}

\author{
Shu-Min Huang ${ }^{1}$, Yu-Wen Lin ${ }^{1}$, Fung-Chang Sung ${ }^{2}$, Chung-Yi $\mathrm{Li}^{3}$, Ming-Fong Chang ${ }^{4}$ and \\ Pei-Chun CHEN ${ }^{2,5}$
}

${ }^{1}$ Department of Public Health, College of Medicine, Fu-Jen Catholic University, ${ }^{2}$ Department of Public Health, China Medical University, ${ }^{3}$ Department of Public Health, College of Medicine, National Cheng-Kung University, ${ }^{4}$ Department of Family Medicine, Sin-Lau Christian Hospital and ${ }^{5}$ Management Office for Health Data, China Medical University Hospital, Taiwan

\begin{abstract}
Occupational Exposure of Dentists to Extremely-low-frequency Magnetic Field: Shu-Min Huang, et al. Department of Public Health, College of Medicine, Fu-Jen Catholic University, TaiwanObjective: To compare occupational exposure to extremely-low-frequency magnetic field (ELF-MF) between dentists practicing in dental clinics and those employed in hospitals. Methods: Thirty-two dentists who worked at clinics $(n=15)$ and 33 dentists employed at hospital dental departments $(n=7)$ voluntarily provided their informed consent to participate in this measurement study. The study dentists were requested to wear an ELF-MF dosimeter for some $3 \mathrm{~h}$ at work to determine their personal exposure. Spot measurements taken at a number of locations in each dental office were used to indicate the work environment exposure level. Additionally, ELF-MF emitted from common dental equipment was also measured. All measurements were performed with EMDEX Lite meters. Results: The average environmental exposure to ELF-MF is higher in clinic dental offices than in hospital dental departments ( 0.55 vs. $0.15 \mu \mathrm{T}, p=0.008$ ). Personal dosimetry showed that on average, clinic dentists spent 35.71 and $19.39 \%$ of their time at exposures above 0.3 and $0.4 \mu \mathrm{T}$ at work, respectively. The corresponding figures for hospital dentists were 19.61 and $13.92 \%$. Additionally, ELF-MF was greater than $0.4 \mu \mathrm{T}$ at $30 \mathrm{~cm}$ from all selected equipment, but the ELF-MF generally diminished as the distance from dental equipment increased. Uultraviolet air sterilization system produced 3 times as much ELF-
\end{abstract}

Received Aug 18, 2010; Accepted Jan 14, 2011

Published online in J-STAGE Feb 17, 2011

Correspondence to: Pei-Chun Chen, Department of Public Health, China Medical University, Taichung 404, Taiwan

(e-mail:eliz0118@gmail.com)

Ming-Fong Chang and Pei-Chun Chen contributed to this article equally.
MF as other dental equipment. Conclusions: This study suggests the possibility of over-exposure of dentists to power frequency ELF-MF. Additionally, certain dental equipment may produce ELF-MF levels greater than $0.4 \mu \mathrm{T}$ in areas where dentists usually work when treating patients.

(J Occup Health 2011; 53: 130-136)

Key words: Dentist, Electromagnetic fields, Extremelylow-frequency, Exposure assessment, Occupational hazards

While epidemiologic evidence tends to indicate an association between residential exposure to extremelylow-frequency magnetic fields (ELF-MF) of 0.4 (microTesla) $\mu \mathrm{T}$ or higher and an increased risk of childhood leukemia, the World Health Organization monograph published in 2007 suggests the possibilities of other health consequences in relation to ELF-MF exposure have not been ruled out ${ }^{1)}$. In 2010, Kroll et al. reported an increased (14\% for leukemia and $34 \%$ for other cancers) but insignificantly estimated relative risk of childhood cancer for each $0.2-\mu \mathrm{T}$ increase in magnetic field ${ }^{2}$. Additionally, a German case-control study in 2010 also looked into the association between parental occupational exposure to ELF-MF and childhood cancer ${ }^{3}$. Moreover, Gobba et al. examined natural killer (NK) cell activity in 52 workers exposed to different levels of ELF-MF during various activities. In higher exposed workers, the study observed a trend of reduced NK activity compared to workers with low exposures, but the difference was not significant ${ }^{4)}$.

Concerns have been raised about occupational ELF-MF exposures of electrical workers and many blue- collar workers, because they are more likely than other workers to use high power electrical equipment ${ }^{5}$. Very few studies have focused on ELF-MF exposures of healthcare workers, who also have potential ELF-MF exposure owing to their 
proximity to certain medical equipment while at work. In a study that assessed low frequency magnetic field exposures in a hospital, a striking variance was found in onsite measurements of magnetic flux density (0.08-6.5 $\mu \mathrm{T})$ and in workers' time-weighted average (TWA) exposures $(0.12-1.04 \mu \mathrm{T})^{6}$. A later study conducted in the pharmacy of a medical center observed average magnetic flux densities between 0.06 and $0.22 \mu \mathrm{T}$, and full-shift TWA exposures of 0.50 and $0.65 \mu \mathrm{T}$ for two pharmacists, respectively ${ }^{7}$. A much lower personal ELFMF exposure, TWA arithmetic means lower than $0.12 \mu \mathrm{T}$, has been reported for other health workers, including physiotherapists, occupational therapists, physicians, nurses and medical radiographers ${ }^{8,9)}$. Possible explanations for the marked discrepancy in field ELF-MF intensities and personal exposure levels of health workers found among studies may include different types and models of medical facilities and equipment operated by healthcare workers. However, biased estimates due to a small sample size, and different strategies of sampling and measurements cannot be entirely ruled out for the reported discrepancies in ELF-MF exposures encountered by health care workers.

Exposure to ELF-MF at work as compared to other activities has been reported to be the highest in daily overall exposure $^{8,9)}$. In an earlier report, Bohay et al. examined some potential sources and intensities of $60 \mathrm{~Hz}$ magnetic fields produced in the dental environment ${ }^{10)}$. The magnetic fields associated with various dental equipment including ultrasonic scalers, amalgamators, composite light curing units, $\mathrm{X}$-ray view boxes and chair lights were measured. The median $60 \mathrm{~Hz}$ field strengths varied among the different types of equipment tested, ranging from 0.12 to $0.22 \mu \mathrm{T}$. In view of recent concerns about the possible effects of magnetic fields, the study by Bohay et al. suggested that exposures be minimized and the concept of prudent avoidance be employed ${ }^{10)}$. Additionally, the UK Adult Brain Tumour Study examined 79 individuals and 25 companies for occupational and non-occupational exposures to ELF-MF. The results showed occupational exposure to be the main determinant of overall exposure. The highest average occupational exposures were found for security officers $(0.78 \mu \mathrm{T})$, secretaries $(0.48 \mu \mathrm{T})$ and dentists $(0.42 \mu \mathrm{T})^{8)}$. Moreover, a recent survey reported ELF-MF of individuals from 117 different occupations. Average exposure was significantly higher at work than at home. The average occupational exposures for dentists (geometric mean $(\mathrm{GM}=0.29 \mu \mathrm{T})$, and dental nurses $(\mathrm{GM}=0.24 \mu \mathrm{T})$ were comparable to the exposures experienced by electricians and electrical fitters $(\mathrm{GM}=0.29$ $\mu \mathrm{T})$, but higher than personal assistants and secretaries $(\mathrm{GM}=0.10 \mu \mathrm{T})$, nurses $(\mathrm{GM}=0.08 \mu \mathrm{T})$, and software professionals $(\mathrm{GM}=0.09 \mu \mathrm{T})$. The survey was also conducted in three dentist's rooms, in the same practice building, and indicated that differing levels of exposure could be explained by alternative positioning of ELF MF sources within the rooms ${ }^{9}$. Despite dentists' potential overexposure to ELF-MF, the ELF-MF intensity in dental settings has not been adequately documented ${ }^{5,6,11)}$. Also, information on dentists' personal exposure to ELF-MF is scarce. This report was therefore conducted to further assess dentists' occupational exposures to ELF-MF.

This study aimed to obtain profiles of ELF-MF exposure of dentists. In Taiwan, dentists usually practice in either hospitals or dental clinics, and these two settings are very dissimilar in area. The floor space of dental clinics is usually limited; therefore the density of dental equipment, sources of ELF-MF, was expected to be higher in clinics than in the dental departments of hospitals. Accordingly, we separately assessed the ELF-MF exposures of dentists in these two settings. Moreover, we not only performed personal ELF-MF exposure measurements using personal dosimeters, but also performed onsite measurements of ELF-MF intensities in work areas where dentists usually treat patients. ELF-MF intensities emitted by some common dental equipment were also determined. Determination of environmental and personal exposures may be useful for addressing dentists' overall occupational exposures to ELF-MF.

\section{Materials and Methods}

\section{Study clinics, hospitals, and dentists}

Between June 2008 and February 2009, a convenient sample of 15 dental clinics and 7 hospitals in northern Taiwan (Taipei City and Taipei County) were solicited for permission to take measurements of dentists' occupational exposures to ELF-MF. We started our measurement work at a clinic just beside the Fu-Jen Catholic University, Taipei, Taiwan. This clinic has been providing dental services for many Fu-Jen students and staff. Upon receiving our request, the first participating dentist referred us to two of his friends, who are also dentists, for measurement trials at their clinics. Our research grant only allowed some 60 dentists to be measured; therefore, we continued the solicitation process until 15 dental clinics had agreed to participate in this study. There were 39 dentists working in these 15 clinics and $32(82 \%)$ agreed to participate in our study. For the hospital setting, we directly contacted 18 hospitals located in the same and neighboring districts as Fu-Jen University, and 11 of them accepted our solicited visits for measurement. Due to difficulties with time schedules, we completed measurements in only 7 hospitals, where 33 (94.3\%) out of 35 dentists were personally measured. Thirty-three dentists employed in the 7 hospitals and 32 dentists from the 15 participating clinics also provided their informed consent to personal dosimetry. The research protocol was reviewed and approved by the Institutional Review Board of Fu-Jen Catholic University, Taipei, Taiwan. 


\section{Personal dosimetry}

The dentists were scheduled to receive some $3 \mathrm{~h}$ of ELF-MF personal dosimetry during a dental treatment session. After a brief instruction regarding how the personal measurements would proceed, each dentist was requested to wear an EMDEX Lite (Enertech Consultants, Campbell, California) on the waist. In addition, one dental assistant helped to complete a preformatted log-book recording the exact time of the start of dental treatment, temporary departures from and returns to the dental office and helped with the removal of the dosimeter when the treatment session was terminated. The dentists were asked to continuously wear the meter during the measurement period. The investigators went to the dental office after the dental session was over to terminate the measurements and retrieved the recorded data from the dosimeters. Measurements were performed with standard EMDEX Lite meters. The measurement accuracy was $\pm 2 \%$ in the frequency band from $40 \mathrm{~Hz}$ to $1 \mathrm{kHz}$; and the sample rate was set at $4 \mathrm{~s}$. All dosimeters were calibrated before use.

\section{Measurement of work environment}

The hospital dental departments were greater than the dental clinics in area. The number of dental chairs in each dental department ranged from 3 to 10 with a mean \pm standard deviation (SD) of $8.43 \pm 3.51$. The corresponding figures for dental clinics were 1 to 6 and $3.13 \pm 1.36$ $(p=0.001)$. An average number of $3.57 \pm 1.72$ hospital dentists and $1.67 \pm 0.98$ clinic dentists $(p=0.005)$ were at work while the measurements were conducted. The majority of clinics $(66 \%)$ and hospital dental departments (94\%) were measured during the summer of 2008 (i.e., June to September, 2008).

We also used EMDEX Lite meters to measure onsite levels of environmental ELF-MF intensity. Treatment areas and waiting areas were measured separately since dental equipment is the main source of ELF-MF in dental offices and is usually located in treatment areas. For each waiting area, we selected the four corners and a number of arbitrarily selected seats for measurements. For each treatment area, in addition to the four corners, we also selected several locations frequently used by dentists for measurements. All measurement locations were $1 \mathrm{~m}$ above the floor and at least $1 \mathrm{~m}$ from the wall to avoid the influence of electrical wiring ${ }^{5}$. Due to the different sizes of the dental offices, the number of measurements varied from 25 to 35 .

\section{Measurement of dental equipment}

Four common pieces of dental equipment, dental chair unit, ultrasonic scaler, composite light curing unit, and ultraviolet air sterilization system, were selected for measurements. The dental chairs was selected because they are an ELF-MF source common to all dentists, and the other three pieces of equipment were selected because of their high consumption of electricity. We performed the measurements following the protocol proposed by the IEEE for surveying controlled environments ${ }^{13)}$. The IEEE standard requires that measurements be collected at various distances (e.g., 0, 10, 30, and $50 \mathrm{~cm}$ ) from the ELF-MF sources. We measured ELF-MF at three locations at different heights above the ground, i.e., 30, 100 and 150 $\mathrm{cm}$, to estimate the exposure of the knee, waist and hand, respectively. At each location, a total of 90 measurements were conducted within six min and a six-minute averaging was calculated. We calculated the spatial averaging derived from the three series of measurements collected over a vertical surface at 30,100 , and $150 \mathrm{~cm}$ above the ground. The spatial averaging was calculated as:

$$
\left[\left(\sum_{i=1}^{\mathrm{n}} \chi_{\mathrm{i}}^{2}\right) / n\right]^{1 / 2}
$$

where $x_{\mathrm{i}}$ is the six-minute average magnetic field intensity ${ }^{14)}$.

\section{Statistical analysis}

Because the hospital dental departments are usually greater than the dental clinics in area, and may have more dental equipment in use simultaneously, dentists employed in hospitals and those who work in clinics may experience different magnetic field intensities. Thus, we decided to perform analyses for hospital dentists and clinic dentists separately.

We first described the characteristics of dentists employed in hospitals and clinics. To account for the asymmetric distribution of magnetic field intensity, we calculated geometric means (GM) and geometric standard deviations (GSD), in addition to arithmetic means (AM) and standard deviations (SD), to better summarize the exposure. The non-parametric Mann-Whitney U test was also used to compare the difference in mean exposures of both environmental and personal measurements between hospital dentists and clinic dentists. A $p$ value less than 0.05 was considered statistically significant. The statistical analysis was performed using SAS 9.1 (SAS Institute, Cary, NC).

\section{Results}

\section{Personal exposure}

More than $60 \%$ of the study dentists were males. Hospital dentists were older than clinic dentists (46.00 \pm 12.64 vs. $37.25 \pm 12.32 \mathrm{yr}$ ). Due to availability, most hospital dentists were measured during morning treatment sessions, whereas a large number of clinic dentists were measured in the evening. The mean duration of personal measurement was similar for hospital and clinic dentists $(3.07 \pm 0.61$ vs. $3.04 \pm 0.80 \mathrm{~h})$ (Table 1$)$.

The average number of measurements were similar for both hospital dentists $(2,766 \pm 552)$ and clinic dentists 
Table 1. Characteristics of the dentists participating in this study

\begin{tabular}{|c|c|c|c|c|}
\hline & \multicolumn{2}{|c|}{ Hospital dentists $(\mathrm{N}=33)$} & \multicolumn{2}{|c|}{ Clinic dentists $(\mathrm{N}=32)$} \\
\hline & $\mathrm{n}$ & $\%$ & $\mathrm{n}$ & $\%$ \\
\hline \multicolumn{5}{|l|}{ Gender } \\
\hline Male & 21 & 63.6 & 21 & 65.6 \\
\hline Female & 12 & 36.4 & 11 & 34.4 \\
\hline \multicolumn{5}{|l|}{ Age } \\
\hline$\leq 29$ & 3 & 9.38 & 11 & 32.14 \\
\hline $30-39$ & 8 & 25.00 & 13 & 39.29 \\
\hline $40-49$ & 6 & 18.75 & 3 & 10.71 \\
\hline $50-59$ & 9 & 28.13 & 2 & 7.14 \\
\hline$\geq 60$ & 6 & 18.75 & 3 & 10.71 \\
\hline Mean \pm SD & \multicolumn{2}{|c|}{$46.00 \pm 12.64$} & \multicolumn{2}{|c|}{$37.25 \pm 12.32$} \\
\hline \multicolumn{5}{|l|}{ Month of measurement } \\
\hline June, 2008 & 3 & 9.09 & 2 & 6.25 \\
\hline July, 2008 & 3 & 9.09 & 2 & 6.25 \\
\hline August, 2008 & 21 & 63.64 & 10 & 31.25 \\
\hline September, 2008 & 4 & 12.12 & 4 & 12.50 \\
\hline October, 2008 & 0 & 0.00 & 1 & 3.13 \\
\hline January, 2009 & 2 & 6.06 & 2 & 6.25 \\
\hline February, 2009 & 0 & 0.00 & 11 & 34.38 \\
\hline \multicolumn{5}{|l|}{ Time of measurement } \\
\hline Morning & 25 & 75.76 & 4 & 12.50 \\
\hline Afternoon & 6 & 18.18 & 8 & 25.00 \\
\hline Evening & 2 & 6.06 & 20 & 62.50 \\
\hline \multicolumn{5}{|l|}{ Duration of measurement } \\
\hline$<2$ & 2 & 6.06 & 2 & 6.25 \\
\hline $2-<3$ & 10 & 30.30 & 15 & 46.88 \\
\hline $3-<3.5$ & 14 & 42.42 & 10 & 31.25 \\
\hline$\geq 3.5$ & 7 & 21.21 & 5 & 15.63 \\
\hline Mean \pm SD & \multicolumn{2}{|c|}{$3.07 \pm 0.61$} & \multicolumn{2}{|c|}{$3.04 \pm 0.80$} \\
\hline
\end{tabular}

$(2,735 \pm 720)$. The minimum and maximum exposures averaged over a treatment session were 0.06 and $0.90 \mu \mathrm{T}$, with an $\mathrm{AM}$ of $0.24 \pm 0.16 \mu \mathrm{T}$ for hospital dentists. The corresponding figures for clinic dentists were $0.05-0.73$ $\mu \mathrm{T}$ and $0.28 \pm 0.16 \mu \mathrm{T}$. The clinic dentists also had a higher mean percentage of time $>0.3 \mu \mathrm{T}$ (35.71 vs. $19.61 \%)$ and a higher mean percentage of time $>0.4 \mu \mathrm{T}$ $(19.39 \%$ vs. $13.92 \%)$ than hospital dentists, but there differences were not statistically significant (Table 2).

\section{Environmental exposure}

At each dental office, 25 to 35 on-site spot measurements were performed, with a mean number of $27.86 \pm 9.89$ and $28.33 \pm 15.29$ for clinics and hospital dental departments, respectively. Table 3 shows the mean environmental ELFMF intensity of dental offices. The minimum and maximum overall ELF-MF in hospital dental departments was 0.07 and $1.30 \mu \mathrm{T}$, respectively. The corresponding figures for clinics were 0.13 and $5.03 \mu \mathrm{T}$. The AM environmental exposure was higher, but not significantly, in clinic dental offices than in hospital dental departments $(0.49 \pm 0.34 \mu \mathrm{T}$ vs. $0.25 \pm 0.18 \mu \mathrm{T}, p=0.113)$. While this analysis was limited to the measurements taken in the treatment areas, we noted a significantly higher AM in clinic dental offices than in hospital dental departments $(0.55 \pm 0.67 \mu \mathrm{T}$ vs. $0.15 \pm 0.08 \mu \mathrm{T}, p=0.008)$. On the other hand, similar ELF-MF exposure levels were noted in the waiting areas (Table 3 ).

\section{Magnetic field emitted from dental equipment}

Probably due to variations in age and model, we noted a substantial variation in maximum (i.e., taken at $0 \mathrm{~cm}$ away from sources) ELF-MF among dental chairs (0.19 to $58.36 \mu \mathrm{T}$ ), with an $\mathrm{AM}$ and $\mathrm{GM}$ of $13.11 \pm 12.40 \mu \mathrm{T}$ and $8.09 \pm 0.33 \mu \mathrm{T}$, respectively. The maximum ELF-MF for ultrasonic scalers also showed a substantial variation, with an $\mathrm{AM}$ and $\mathrm{GM}$ of $48.25 \pm 5.91 \mu \mathrm{T}$ and $7.01 \pm 1.96$ $\mu \mathrm{T}$, respectively. The AM/GM of maximum ELF-MF for composite light curing units and ultraviolet air sterilization systems were $19.15 \pm 16.93 \mu \mathrm{T} / 5.43 \pm 1.75 \mu \mathrm{T}$ and 23.77 $\pm 11.87 \mu \mathrm{T} / 22.23 \pm 0.17 \mu \mathrm{T}$, respectively. But these figures were based on only 3 composite light curing units and 2 ultraviolet air sterilization machines (Table 4).

Table 4 also demonstrates the clear decreasing trend in 
Table 2. Magnetic field intensity $(\mu \mathrm{T})$ exposure of dentists at the time of performing dental treatment

\begin{tabular}{|c|c|c|c|}
\hline Statistic $^{\mathrm{a}}$ & Hospital dentists $(\mathrm{N}=33)$ & Clinic dentists $(\mathrm{N}=32)$ & $p$ value \\
\hline \multicolumn{4}{|l|}{ Average exposure } \\
\hline Min. - Max. & $0.06-0.90$ & $0.05-0.73$ & \multirow{3}{*}{$0.283^{\mathrm{b}}$} \\
\hline $\mathrm{AM} \pm \mathrm{SD}$ & $0.24 \pm 0.16$ & $0.28 \pm 0.16$ & \\
\hline $\mathrm{GM} \pm \mathrm{GSD}$ & $0.20 \pm 0.18$ & $0.23 \pm 0.19$ & \\
\hline \multicolumn{4}{|l|}{$\%$ of time $>0.3 \mu \mathrm{T}$} \\
\hline Min.-Max. & $0.00-91.71$ & $0.20-99.96$ & \multirow[b]{2}{*}{$0.064^{\mathrm{b}}$} \\
\hline $\mathrm{AM} \pm \mathrm{SD}$ & $19.61 \pm 28.89$ & $35.71 \pm 38.87$ & \\
\hline \multicolumn{4}{|l|}{$\%$ of time $>0.4 \mu \mathrm{T}$} \\
\hline Min.-Max. & $0.00-88.09$ & $0.00-98.88$ & \\
\hline $\mathrm{AM} \pm \mathrm{SD}$ & $13.92 \pm 25.90$ & $19.39 \pm 31.70$ & $0.448^{\mathrm{b}}$ \\
\hline
\end{tabular}

a $\mathrm{AM}=$ arithmetic mean; $\mathrm{SD}=$ standard deviation; $\mathrm{GM}=$ geometric mean; GSD=geometric standard deviation.

${ }^{\mathrm{b}}$ Mann-Whitney U Test.

Table 3. Mean environmental magnetic field intensity $(\mu \mathrm{T})$ of dental offices

\begin{tabular}{cccc}
\hline Statistic & Hospitals & Clinics & \\
\cline { 2 - 3 } & $\mathrm{n}=7$ & $\mathrm{n}=15$ & \\
\hline Overall & & & \\
Min. - Max. & $0.07-1.30$ & $0.13-5.03$ & \multirow{2}{*}{0.113} \\
AM \pm SD & $0.25 \pm 0.18$ & $0.49 \pm 0.34$ & \\
GM \pm GSD & $0.18 \pm 0.12$ & $0.25 \pm 0.14$ & \\
Treatment area & & & \\
Min. - Max. & $0.08-0.34$ & $0.14-2.51$ & \\
AM \pm SD & $0.15 \pm 0.08$ & $0.55 \pm 0.67$ & \\
GM \pm GSD & $0.14 \pm 0.07$ & $0.27 \pm 0.13$ & \\
Waiting area & & & \\
Min. - Max. & $0.17-0.55$ & $0.22-0.14$ & \\
AM \pm SD & $0.35 \pm 0.51$ & $0.31 \pm 0.18$ & \\
GM \pm GSD & $0.30 \pm 0.43$ & $0.29 \pm 0.17$ & \\
\hline
\end{tabular}

a $\mathrm{AM}=$ arithmetic mean; $\mathrm{SD}=$ standard deviation; $\mathrm{GM}=$ geometric mean; $\mathrm{GSD}=$ geometric standard deviation. ' ${ }^{\mathrm{b}}$ Mann-Whitney U Test.

ELF-MF as the distance from dental equipment increased. The AM/GM for dental chair units was $0.46 \pm 0.37 \mu \mathrm{T} /$ $0.36 \pm 0.20 \mu \mathrm{T}$ at $30 \mathrm{~cm}$, which is the distance dentists usually keep while treating patients. The corresponding figures for ultrasonic scalers were similar at $0.41 \pm 0.37$ $\mu \mathrm{T} / 0.27 \pm 0.33 \mu \mathrm{T}$, but were greater for composite light curing units $(0.85 \pm 1.01 \mu \mathrm{T} / 0.45 \pm 0.58 \mu \mathrm{T})$ and ultraviolet air sterilization systems $(1.51 \pm 0.78 \mu \mathrm{T} / 1.41$ $\pm 0.17 \mu \mathrm{T})$.

\section{Discussion}

This study revealed that on average, during treatment sessions, hospital dentists spent 20 and $14 \%$ of time at levels of ELF-MF exposure in excess of 0.3 and $0.4 \mu \mathrm{T}$, respectively; the corresponding figures for clinic dentists were even higher. Moreover, the average ELF-MF intensity was greater than $0.4 \mu \mathrm{T}$ at $30 \mathrm{~cm}$ from all dental equipment, a distance that dentists usually keep when performing treatments, suggesting potential overexposure of ELF-MF for dentists while operating various dental equipment.

Previous occupational studies assessed ELF-MF exposures associated with various jobs including dentists and dental nurses, using personal dosimeters ${ }^{8-11)}$. A UK study ${ }^{8)}$ reported that dentists had the third highest mean exposure (time-weighted average [TWA], $0.42 \mu \mathrm{T}$ ) among various job titles, next to security officer $(0.78 \mu \mathrm{T})$ and secretaries $(0.48 \mu \mathrm{T})$. Workers with other job titles had mean exposures not higher than $0.20 \mu \mathrm{T}$ except for dental nurses $(0.30 \mu \mathrm{T})$. Personal ELF-MF exposure of dentists in our study was lower than that of UK dentists but higher than that associated with most occupational titles.

Levels of ELF-MF in dental environments have been measured by type of and distance from dental equipment 
Table 4. Magnetic field intensity $(\mu \mathrm{T})$ exposure of dentists at the time of performing dental treatment

\begin{tabular}{|c|c|c|c|c|c|}
\hline & & & ance from mag & source $(\mathrm{cm})$ & \\
\hline & $\mathrm{n}$ & 0 & 10 & 30 & 50 \\
\hline Dental chair unit & 29 & & & & \\
\hline Min. & & 0.19 & 0.15 & 0.14 & 0.04 \\
\hline Max. & & 58.36 & 7.19 & 1.90 & 1.52 \\
\hline Median & & 9.63 & 2.14 & 0.38 & 0.31 \\
\hline $\mathrm{AM} \pm \mathrm{SD}$ & & $13.11 \pm 12.40$ & $2.48 \pm 1.87$ & $0.46 \pm 0.37$ & $0.32 \pm 0.31$ \\
\hline $\mathrm{GM} \pm \mathrm{GSD}$ & & $8.09 \pm 0.33$ & $1.79 \pm 0.25$ & $0.36 \pm 0.20$ & $0.23 \pm 0.24$ \\
\hline Ultrasonic scaler & 15 & & & & \\
\hline Min. & & 0.13 & 0.06 & 0.05 & 0.05 \\
\hline Max. & & 113.12 & 11.52 & 0.97 & 0.28 \\
\hline Median & & 14.68 & 2.97 & 0.39 & 0.15 \\
\hline $\mathrm{AM} \pm \mathrm{SD}$ & & $48.25 \pm 5.91$ & $4.4 \pm 4.78$ & $0.41 \pm 0.37$ & $0.16 \pm 0.10$ \\
\hline $\mathrm{GM} \pm \mathrm{GSD}$ & & $7.01 \pm 1.96$ & $1.61 \pm 0.82$ & $0.27 \pm 0.33$ & $0.13 \pm 0.21$ \\
\hline Composite light curing unit ${ }^{a}$ & 3 & & & & \\
\hline Min. & & 0.20 & 1.20 & 0.13 & 0.06 \\
\hline Max. & & 32.80 & 11.24 & 1.56 & 0.25 \\
\hline Median & & 24.45 & 6.22 & 0.85 & 0.16 \\
\hline $\mathrm{AM} \pm \mathrm{SD}$ & & $19.15 \pm 16.93$ & $6.22 \pm 7.01$ & $0.85 \pm 1.01$ & $0.16 \pm 0.13$ \\
\hline $\mathrm{GM} \pm \mathrm{GSD}$ & & $5.43 \pm 1.75$ & $3.67 \pm 0.49$ & $0.45 \pm 0.58$ & $0.12 \pm 0.27$ \\
\hline Ultraviolet air sterilization system ${ }^{\text {b }}$ & 2 & & & & \\
\hline Min. & & 15.37 & 4.84 & 0.96 & 0.61 \\
\hline Max. & & 32.16 & 16.08 & 2.06 & 1.34 \\
\hline Median & & 23.77 & 10.46 & 1.51 & 0.98 \\
\hline $\mathrm{AM} \pm \mathrm{SD}$ & & $23.77 \pm 11.87$ & $10.46 \pm 7.95$ & $1.51 \pm 0.78$ & $0.98 \pm 0.52$ \\
\hline $\mathrm{GM} \pm \mathrm{GSD}$ & & $22.23 \pm 0.17$ & $8.82 \pm 0.23$ & $1.41 \pm 0.17$ & $0.90 \pm 0.18$ \\
\hline
\end{tabular}

${ }^{\mathrm{a}}$ Ortholux XT Visible Light Curing Unit 3M UNITEK $(\mathrm{n}=2)$; Elipar ${ }^{\mathrm{TM}}$ S10 LED Curing Light, 3M ESPE (n=1). ${ }^{\mathrm{b}}$ SAMPO MEDICAL $\mathrm{T}-378(\mathrm{n}=2)$.

in a few studies ${ }^{10,15)}$. One prior study ${ }^{10)}$ evaluated the ELF-MF intensities produced by ultrasonic scalers and light curing units, which were also measured in our study. In line with our observations, the ELF-MF levels were higher than $0.4 \mu \mathrm{T}$ at $30 \mathrm{~cm}$ from both types of equipment when they were turned on. The intensity was generally consistent with previous findings ${ }^{10}$. It is noteworthy that in our study, the ELF-MF level at $30 \mathrm{~cm}$ from ultraviolet air sterilization systems was $1.41 \mu \mathrm{T}$, more than 3 times higher than that from other dental equipment; the ELF-MF intensity moderately reduced to $0.9 \mu \mathrm{T}$ at $50 \mathrm{~cm}$. Although limited in sample size, our observations suggest that dentists should avoid long-term operation of ultraviolet air sterilization systems and stay away from them in order to limit exposure to ELF-MF.

In this study, exposure levels obtained from environmental measurements were similar to those obtained in personal dosimetry of hospital dentists (AM: 0.25 vs. $0.24 \mu \mathrm{T}$ ), and, the environmental ELF-MF level at dental offices was much higher than the level obtained in personal dosimetry of clinic dentists (AM: 0.49 vs. 0.28 $\mu \mathrm{T})$. One of possible explanations is the difference in area between hospital dental departments and clinic dental offices. Dental clinic offices are usually limited in space and the environmental ELF-MF level is therefore more likely affected by sources of ELF-MF, such as TVs, computers, printers, air-conditioners and fans ${ }^{16)}$. Air conditioners are commonly used in summer in Taiwan, and the highest levels of ELF-MF have been reported to occur in this season ${ }^{17)}$. The much higher environmental ELF-MF intensity observed in dental clinic offices, relative to exposure levels of personal dosimetry of clinic dentists, might be because most of our survey was performed in summer. Moreover, we did not take into account the temporal variation of ELF-MF intensities in dental offices. Unlike the 3-hour personal dosimetry performed for dentists, we performed only short-term field survey of environmental exposure in which 25 to 35 measurements of magnetic flux densities in each dental office were made. Previous studies have reported temporal variation in ELFMF intensity during 8-hour ${ }^{7)}$ and 24-hour periods ${ }^{18)}$. Failure to take into account the temporal variation in ELFMF may have resulted in misrepresentation of the true environmental exposure in dental offices; however, the 
exposure error would not have been systematically different between hospital and dental office environments. The temporal variability might also partly explain the discrepancy between levels of personal exposure and the environmental intensity of ELF-MF in the dental clinics. Moreover, our study findings were based on a volunteer sample of dentists, and the sample size was not considered large enough to assure external validity, which limits the generalizibility of our study findings.

The other limitation to our study was related to the selection of places for measurement and the measurement time periods were not identical in the dental offices involved in our study, mainly due to practical reasons. All the hospital dental departments and clinical dental offices were solicited to participate in this study, and they were different in size and interior design. As such, although we used a standardized measurement protocol, we were unable to completely follow the measurement protocol in the field, and could only take the measurements at places where we were allowed access.

Despite controversy about the possible adverse health effect to adults of ELF-MF exposure, reducing occupational ELF-MF exposure of dentists is of importance as their exposure is on a daily basis. Equipment such as ultraviolet air sterilization systems, which emit high ELF-MF levels and are not a built-in element of the main dental unit, can be placed away from dentists' working area. In addition, ELF-MF magnitude has been reported to be dissimilar among different models and types of dental instruments ${ }^{15)}$. Limitation of utilization or appropriate allocation of the models that emit high ELF-MF intensity should be considered when setting up dental work stations in order to limit dentists' exposure to ELF-MF. In conclusion, it is possible for dentists to encounter overexposure to ELFMF while treating their patients. Prudent avoidance to such exposures can be achieved by appropriate positioning of dental equipment in the office.

Acknowledgments: This project was supported by grants from the Taiwan National Science Council (NSC 98-2314-B-227-001-MY2), and Taiwan Department of Health (DOH100-TD-B-111-004/DOH100TD-C-111-005). We are also grateful to the study participants.

\section{References}

1) World Health Organization. Extremely Low Frequency Fields. Environmental Health Criteria 238. [Online]. 2007 [cited 2010 Aug 17]; Available from: URL: http:// www.who.int/peh-emf/publications/elf_ehc/en/index. html

2) Kroll ME, Swanson J, Vincent TJ, Draper GJ. Childhood cancer and magnetic fields from high-voltage power lines in England and Wales: a case-control study. Br J Cancer 2010; 103: 1122-7.
3) Hug K, Grize L, Seidler A, Kaatsch P, Schüz J. Parental occupational exposure to extremely low frequency magnetic fields and childhood cancer: a German casecontrol study. Am J Epidemiol 2010; 171: 27-35.

4) Gobba F, Bargellini A, Scaringi M, Bravo G, Borella P. Extremely low frequency-magnetic fields (ELF-EMF) occupational exposure and natural killer activity in peripheral blood lymphocytes. Sci Total Environ 2009; 407: 1218-23.

5) Bracken TD. Exposure assessment for power frequency electric and magnetic fields. Am Ind Hyg Assoc J 1993; 54: 165-77.

6) Philips KL, Morandi MT, Oehme D, Cloutier PA. Occupational exposure to low frequency magnetic fields in health care facilities. Am Ind Hyg Assoc J 1995; 56: 677-85.

7) Li CY, Lin RS, Wu CH, Sung FC. Occupational exposures of pharmacists and pharmaceutical assistants to $60 \mathrm{~Hz}$ magnetic fields. Ind Health 2000 ; 38: 413-9.

8) van Tongeren $\mathrm{M}$, Mee $\mathrm{T}$, Whatmough $\mathrm{P}$, et al. Assessing occupational and domestic ELF magnetic field exposure in the UK adult brain tumour study: results of a feasibility study. Radiat Prot Dosimetry 2004; 108: 227-36.

9) Mee T, Whatmough P, Broad L, et al. Occupational exposure of UK adults to extremely low frequency magnetic fields. Occup Environ Med 2009; 66: 61927.

10) Bohay RN, Bencak J, Kavaliers M, Maclean D. A survey of magnetic fields in the dental operatory. J Can Dent Assoc 1994; 60: 835-40.

11) Forssén UM, Mezei G, Nise G, Feychting M. Occupational magnetic field exposure among women in Stockholm County, Sweden. Occup Environ Med 2004; 61: 594-602.

12) Moriyama K, Yoshitomi K. Apartment electrical wiring: a cause of extremely low frequency magnetic field exposure in residential areas. Bioelectromagnetics 2005; 26: $238-41$.

13) IEEE. IEEE recommended practice for the measurement of potentially hazardous electromagnetic fields-RF and microwave (C95.3-1991), IEEE, New York, 1992.

14) Li CY, Feng CK. An evaluation of radio frequency exposure from therapeutic diathermy equipment. Industrial Health 1999; 37: 465-8.

15) Buković D Jr, Carek V, Durek D, Kuna T, Keros J. Measurement of magnetic field in dentistry. Coll Antropol 2000; 24 (Suppl 1): 85-9.

16) Mezei G, Kheifets LI, Nelson LM, Mills KM, Iriye R, Kelsey JL. Household appliance use and residential exposure to $60-\mathrm{Hz}$ magnetic fields. J Expo Anal Environ Epidemiol 2001; 11: 41-9.

17) Saito T, Nitta H, Kubo O, et al. Power-frequency magnetic fields and childhood brain tumors: a casecontrol study in Japan. J Epidemiol 2010; 20: 54-61.

18) Paniagua JM, Jiménez A, Rufo M, Antolín A. Exposure assessment of ELF magnetic fields in urban environments in Extremadura (Spain). Bioelectromagnetics 2004; 25 : $58-62$. 